\title{
Psicoterapias intensivas prolongadas ambulatorias para niños y adolescentes con trastorno mental grave
}

\section{Long-term intensive ambulatory psychotherapies for children and adolescents with severe mental disorder}

\author{
Diego Padilla Torres ${ }^{1}$, Eloy Francisco Albaladejo Gutiérrez ${ }^{2}$ e Inmaculada Palanca Maresca ${ }^{1}$ \\ ${ }^{1}$ Hospital Universitario Puerta de Hierro-Majadahonda, España \\ ${ }^{2}$ AGCPSM Hospital 12 Octubre de Madrid, España
}

\begin{abstract}
Resumen: Dentro del Sistema Nacional de Salud (SNS), la prestación de psicoterapia es una de las técnicas aplicadas tanto por el médico especialista en Psiquiatría de la Infancia y Adolescencia como por el especialista en Psicología Clínica de la Infancia y Adolescencia. El objetivo de este trabajo es exponer la importancia de la psicoterapia intensiva a largo plazo en el tratamiento de niños y jóvenes con trastornos mentales graves.

Palabras clave: psicoterapia prolongada e intensiva, tratamientos psicoterapéuticos, niños, adolescentes, trastorno mental grave infanto-juvenil, costes económicos, psiquiatría de la infancia y adolescencia, psicología clínica de la infancia y adolescencia.

Abstract: Within the Spanish National Health System (SNS), psychotherapy is one of the techniques used by both physicians specializing in Childhood and Adolescent Psychiatry and by specialists in Clinical Childhood and Adolescence Psychology. The present study describes the importance of long-term intensive psychotherapy in the treatment of children and young people with severe mental disorders.
\end{abstract}

\footnotetext{
Padilla Torres, Diego es Psicólogo Clínico Infancia y Adolescencia. CSM Majadahonda. Hospital Universitario Puerta de Hierro-Majadahonda. Madrid.

Albaladejo Gutiérrez, Eloy Francisco es Psiquiatra Infancia y Adolescencia. Médico Psiquiatra-Coordinador sección infantil en Hospital de Día Infanto-Juvenil Pradera de San Isidro. AGCPSM Hospital 12 Octubre de Madrid.

Palanca Maresca, Inmaculada es Psiquiatra del niño y el adolescente. Responsable de Unidad de Psiquiatría y Psicologia Clínica del Niño y el Adolescente. Servicio de Psiquiatría. Hospital Universitario Puerta de Hierro-Majadahonda. Madrid.

Para citar este artículo: Padilla Torres, D., Albaladejo Gutiérrez, E. F. y Palanca Maresca, I. (2021). Psicoterapias intensivas prolongadas ambulatorias para niños y adolescentes con trastorno mental grave. Clínica Contemporánea, 12(2), Artículo e12. https://doi.org/10.5093/cc2021a11

La correspondencia sobre este artículo debe enviarse al primer autor al Hospital. Universitario Puerta de Hierro Majadahonda. Psiquiatría y Psicología Clínica de la Infancia y Adolescencia.Calle Joaquín Rodrigo, 1. Majadahonda, Madrid 28222. E-mail: diego.padilla@salud.madrid.org
} 
Keywords: prolonged and intensive psychotherapy, psychotherapeutic treatments, children, adolescents, severe child-adolescent mental disorder, economic costs, childhood and adolescent psychiatry, clinical childhood and adolescence psychology

La patología mental en la población infanto-juvenil tiene una elevada prevalencia: 1 joven de cada 10 padece un problema de salud mental. El $75 \%$ de los adultos con un problema de salud mental diagnosticable experimentan los primeros síntomas antes de 24 años; el 50\% de la patología adulta comienza en la infancia (Kessler et al., 2005; McGorry, Purcell et al., 2007). Entre el 10 y el 20\% de los menores de 0 a 17 años tiene un diagnóstico de problemas de salud mental, y el suicidio es la tercera causa de muerte en adolescentes (Navarro-Gómez, 2017). En niños y adolescentes con trastorno mental, este conlleva una pérdida de entre 4 y 10 años por discapacidad (OMS, 2018).

Estudios sobre encuestas de salud mental realizados en población infanto-juvenil, recogen que el $8.5 \%$ de los niños tiene problemas emocionales, $6.7 \%$ problemas conductuales, $10.2 \%$ hiperactividad, $7.7 \%$ problemas en las relaciones con iguales y $0.7 \%$ problemas en la conducta prosocial (Basterra, 2016).

La prevalencia de trastornos mentales es del $10 \%$ para menores de 18 años, entre un $4 \%$ y $6 \%$ son de trastorno mental grave infanto-juvenil (TMG-IJ) (Kessler et al., 2005). Los trastornos mentales graves en la infancia y adolescencia se definen por ser patologías con un diagnóstico grave (trastornos psicóticos, trastornos afectivos, autismo, trastornos de ansiedad, de conducta, de personalidad) de aparición temprana, con pronóstico de gravedad clínica, comorbilidad y factores sociales asociados (Fernández Liria y Gómez Beneyto, 2009; Mollejo Aparicio, 2012). Además sabemos que la infancia, la adolescencia y la juventud son períodos críticos del neurodesarrollo más productivo (Arnett y Arnett, 2007; Paus et al., 2008) cuya desviación o alteración tendrá grandes consecuencias en la edad adulta y en generaciones posteriores (Patton et al., 2018). Si tomamos como ejemplo el trastorno del espectro autista (TEA), la prevalencia oscila entre 1/66 y 1/150 casos por nacido vivo (Volkmar et al., 2014) lo que, junto con la alta prevalencia de discapacidad asociada y la altísima frecuencia de comorbilidad con problemas de salud mental asociados (Simonoff et al., 2008), convierte la atención a los TEA en un problema asistencial prioritario.

La prevalencia recogida en el último Plan de Salud Mental de la Comunidad de Madrid (Oficina Regional de Coordinación de Salud Mental, 2018) es de un 8.5\% de problemas emocionales, $10.2 \%$ de trastorno por déficit de atención e hiperactividad y $0.01 \%$ de TEA. No obstante, en función de los estudios las tasas pueden estar aumentadas o disminuidas, pero por ejemplo en otros estudios las tasas depresión en niños y adolescentes están entre 10 y 14\% (Aláez et al., 2000; Bragado et al., 1999) en Madrid. Por lo que tan solo en la Comunidad de Madrid, con una población menor de 18 años de 1291613 existirían alrededor 109787 niños con problemas emocionales, 131744 con problemas de inatención e hiperactividad y unos 129.16 tendrían trastornos del espectro autista. Por lo que 129161 tendrán un trastorno mental (10\% de la población) y 51666 presentarán un TMG-IJ. Los recursos existentes no pueden dar cabida a dicha demanda.

Los costes directos e indirectos de las patologías mentales en los niños son altos. Ante las carencias del sistema sanitario público, muchos padres tiene que faltar al trabajo o acudir a la red privada. Además, los niños tienen problemas de salud mental, tienen más posibilidades de fracaso escolar y social, menos posibilidades de encontrar trabajo en el futuro, así como alta probabilidad de permanecer como usuarios de la red de salud mental. A falta de datos claros y concretos en España, en otros países del entorno como Inglaterra, se estima que el gasto en salud mental de niños entre 5 y 15 años es de $£ 1803$ por niño y año. En niños con diagnóstico de TDAH puede ser de $£ 3108$ niño/año, seguido de trastornos de conducta de $£ 1856$, afectivos de $£ 1165$ y casi de $£ 3000$ para el caso de los autistas (Beecham, 2014). Igualmente, el gasto sanitario y social se ha visto como tiene consecuencias no sólo en este grupo etario (Marc Suhrcke et al., 2007), sino que tiene consecuencias en la adultez. Diversos estudios señalan que los trastornos de inicio en la infancia no tratados se mantienen en la etapa adulta generando costes en esta edad. En estudios con trastornos de conducta, seguidos a lo largo de 28 años, se encontró que los costos para los adultos eran 10 veces mayores para los que presentaban trastorno 
de conducta en la infancia, ya que el trastorno tiende a persistir y se acumulan actos delictivos, violentos y judiciales (Scott et al., 2002).

Si además del gasto sanitario se suman los gastos que se asocian en relación con intervenciones de instituciones judiciales, sociales y educativas o de asociaciones, el gasto aumenta. Por lo que para un niño con un diagnóstico de problemas a nivel emocional y de conducta grave el gasto en un año es, para el sistema de salud de 3499 euros, el coste a nivel de servicios sociales 32999 euros, a nivel del sistema educativo 24587 euros, del sistema judicial 3235 euros y en servicios voluntarios en asociaciones 655 euros (Suhrcke et al., 2008).

Además del gasto económico sabemos que los trastornos mentales comunes en la infancia y adolescencia están entre las cinco primeras causas de pérdida de años de vida ajustados en función de la discapacidad (AVAD) en los adolescentes (Consejo General de la Psicología de España, 2019). En el caso de los TMG-IJ, la carga de los padres en el cuidado de sus hijos, la pérdida de tiempo en el trabajo, las consecuencias emocionales en los padres, las pérdidas de las posibilidades de promoción en el trabajo, la restricción en las relaciones sociales, son algunas de las consecuencias del cuidado de los hijos con TMG-IJ, además de las propias consecuencias directas para la vida de los pacientes, que afecta al bienestar emocional de los padres.

En España, no tenemos datos sobre los gastos directos e indirectos, ni datos en relación a la red sanitaria necesaria para el abordaje del TMG-IJ, aspecto que contrasta con lo señalado por aportaciones clínicas y teóricas que constatan que para la salud es necesario una abordaje lo más pronto posible así como en TMG-IJ (Laffan y Faulconbridge, 2016; Faulconbridge et al., 2015) intervenciones tempranas e intensivas que generan consecuencias positivas tanto en el área de salud, como en el de educación, justicia y bienestar social (Scott et al., 2002; Skokauskas et al., 2018).

A día de hoy, llama la atención que no exista un plan de actuación homogéneo en el Estado español, y que los planes de intervención de TMG-IJ no estén bien insertados en las políticas sanitarias, ni se cuente con recursos económicos y humanos especializados. En estudios realizados en nuestros país sobre el TMG-IJ, se recoge la necesidad de poder articular programas de mayor atención en los centros de salud mental infanto-juvenil y en los hospitales de día (Lasa-Zulueta et al., 2014) así como se constatan las deficiencias en el abordaje del TMG-IJ en nuestro país.

En búsqueda de soluciones efectivas, que promuevan un menor gasto sanitario en la población infanto-juvenil, y que reduzcan los gastos futuros en la población adulta relacionados con problemas no resueltos efectivamente en la infancia, consideramos que la psicoterapia intensiva y prolongada puede ser una herramienta que dé respuesta a ello, psicoterapia llevada a cabo dentro de los centros de salud mental ambulatorios comunitarios.

\section{Psicoterapia en el trastorno mental grave infanto-juvenil}

El Plan Integral de Salud Mental 2013-2020 de la Organización Mundial de la Salud (OMS, 2013), indica que "Los niños y adolescentes con trastornos mentales deben ser objeto de intervenciones tempranas científicamente contrastadas, de carácter no farmacológico, evitando la hospitalización y la medicalización”. Además, el Plan Estratégico Nacional de Infancia y Adolescencia (Observatorio de la Infancia, 2013) señala la necesidad de un aumento de las intervenciones psicológicas y sociales. Estas indicaciones contrastan con la realidad de los datos ofrecidos que señalan el aumento de las intervenciones farmacológicas. El Plan de Infancia y Adolescencia de la Comunidad de Madrid (2017-2021), establece diferentes líneas de acción dirigidas a desarrollar Programas de Continuidad de Cuidados para TMG-IJ en coordinación con la Consejería de Sanidad (Acción 103) (Dirección General de la Familia y el Menor, 2017, p. 102) y también al desarrollo de programas psicoterapéuticos para niños y niñas que necesitan ayuda especializada (Acción 132) (Dirección General de la Familia y el Menor, 2017, p. 107).

La psicoterapia es fundamental en el TMG-IJ, (Bardón, 2012; Mollejo Aparicio, 2012). El abordaje, para casos tan complicados, es necesario que sea llevado a cabo por profesionales con titulaciones oficiales de Psiquiatría y Psicología Clínica, a la espera del reconocimiento de las especialidades de Psiquiatría de la Infancia y Adolescencia y de Psicología Clínica de Infancia y Adolescencia. 
Actualmente la psicoterapia de niños y adolescentes cuenta con un cuerpo de evidencia empírica cada vez mayor en diferentes patologías (Jones et al., 2019; Knapp et al., 2016; Midgley et al., 2018; Miller et al., 2013; O’Brien et al., 2013; Pérez Álvarez, 2010; Shirk et al., 2011). La Federación Española Asociaciones de Psicoterapia (FEAP) define la psicoterapia como

... todo tratamiento de naturaleza psicológica que, a partir de manifestaciones psíquicas o físicas de sufrimiento humano, promueve el logro de cambios o modificaciones en el comportamiento, relaciones, cogniciones, adaptación al entorno, salud física y psíquica, integración de la identidad psicológica y en general el mejor equilibrio y bienestar biopsico-social de las personas y grupos tales como la pareja o la familia. (FEAP, 2007, p. 2)

Desde otro punto de vista, entendemos la psicoterapia como

... tratamiento interpersonal que a) se basa en principios psicológicos, b) implica un terapeuta formado y un paciente que busca ayuda para un trastorno, problema o queja, c) entendido que el terapeuta puede remediarlo, y d) se adapta a un paciente particular y a su trastorno o queja. (Wampold e Imel, 2015, p. 37)

Para Shirk (1998), autor más centrado en psicoterapia infanto-juvenil, la psicoterapia puede definirse en tres dimensiones: a) estructura: grado en que la psicoterapia es dirigida por el psicoterapeuta y por el niño; b) medio: continuum de intercambios entre el niño y el terapeuta que van de la conversación al juego; y c) funciones comunicativas: un continuum de comunicaciones entre terapeuta y niño que van desde la interpretación que analiza el significado de la conducta del niño o comunicación que proporciona una experiencia emocional correctiva en un medio de apoyo (Shirk, 1988).

La psicoterapia está basada en una relación interpersonal donde la palabra, las propias emociones del terapeuta, junto con otras acciones, entran en interacción con las emociones, pensamientos y acciones del niño provocando cambios en su mundo interno y externo que irán en la dirección de avanzar en su desarrollo de forma positiva y constructiva.

En el caso de pacientes graves, desde nuestra experiencia psicoterapéutica, observamos que la psicoterapia está impregnada de una relación intensa, donde el niño va a trasmitir al clínico mensajes encriptados con su juego, con su lenguaje, con sus síntomas, mensajes que desde la teoría y la práctica se podrán ir descifrando, ayudando a la persona a afrontar los avatares de su trastorno.

Por lo tanto, la práctica en psicoterapia con niños y adolescentes va a necesitar de competencias emocionales y relaciones en el psicoterapeuta de niños y adolescentes, diferente al terapeuta de adultos, y con conocimientos teóricos y técnicos distintos al profesional de adultos. Conocimientos teóricos sobre teorías del desarrollo, psicología evolutiva, psicopatología evolutiva, o las formas de presentación psiquiátrica de los trastornos mentales en la infancia y adolescencia, pero también sobre la red y funcionamiento de salud mental, habilidades lingüísticas y de juego, procesos transdiagnósticos, así como una visión integradora, holística, humana y contextual de la psicoterapia (Pérez-Alvarez, 2019).

Creemos por tanto que la psicoterapia, para niños y adolescentes que presentan una patología mental grave y que en la evaluación se indique que pueden beneficiarse de ella, necesita de unas condiciones específicas. Tiene que ser ambulatoria, intensiva y prolongada en el tiempo. Consideramos intensiva, porque tiene que ser una psicoterapia centrada en el mundo emocional del niño, y centrada en un vínculo terapéutico con el paciente. Y prolongada, para patologías graves menos de un año de tratamiento no es suficiente para poder recuperar funciones perdidas en los niños (Fonagy et al., 2017; Green, 2009; Shedler, 2010; Trowell et al., 2007).

Este encuadre psicoterapéutico tiene que ir acompañado por entrevistas con los padres e intervenciones en otros contextos como el colegio u otros grupos o lugares de intervención o asociaciones a las que acuda. Además, consideramos que lo mejor es que sea ambulatoria, es decir, que se lleve a cabo en el centro de salud mental infanto-juvenil, por varias razones. Por la cercanía a su domicilio, que previene la interferencia con la vida cotidiana del coste en tiempo y dinero de los desplazamientos, y por ser el centro de cuidados sobre el que se vertebra y planifica la atención coordinada de distintos profesionales dentro del equipo multidisciplinar infanto-juvenil. 
Nuestra consideración sobre la psicoterapia se basa en los principios de la psicoterapia de niños y jóvenes y en dicho campo de conocimiento. No obstante, queremos remarcar el papel que juega la psicoterapia en el TMG-IJ, como una prestación a garantizar en el centro de salud mental ambulatorio. En el apartado siguiente profundizamos en la psicoterapia intensiva ambulatoria.

\section{Psicoterapia intensiva ambulatoria para trastorno mental grave infanto juvenil}

La psicoterapia intensiva es un tratamiento altamente especializado, que se aplica por psicoterapeutas (médicos especialistas en psiquiatría o psicólogos clínicos) con entrenamiento específico en psicoterapia en niños y adolescentes. Modalidad terapéutica que se va a dar de forma estructurada durante un tiempo prolongado. Es un tratamiento largo, porque el paciente es atendido al menos un año, e intensivo porque es visto al menos una vez a la semana, y en algunos momentos de crisis, pueden ser puntualmente atendidos dos veces por semana (ACPCAP, 2019), llevando simultáneamente intervenciones con los padres, y coordinaciones con el colegio u otros profesionales (p. ej. pediatras, logopedas).

Los pacientes que se deriven al tratamiento psicoterapéutico intensivo, serán aquellos que en la evaluación multidisciplinar se valore que presentan un trastorno mental grave en la infancia y adolescencia y que se pueden beneficiar de un tratamiento psicoterapéutico, estructurado y a largo plazo. No incluimos los trastornos autistas ya que necesitan un trabajo en paralelo de profundización y reflexión, pero para poner de relieve la importancia de la psicoterapia intensiva y larga, nos vamos a centrar en ejemplos de psicoterapias para trastornos no autistas, pero perfectamente los tratamientos de los trastornos del espectro autista servirían también de ejemplo.

La psicoterapia prolongada e intensiva se proporcionará tras un proceso de decisión clínica compartida entre los profesionales del equipo que atienden el caso, dentro del proceso de intervención en trastorno mental grave infantil y que debe estar incluido en los programas o proceso de intervención en trastorno mental grave de cada servicio de salud mental. Por lo que en cada servicio será el equipo de TMG-IJ, el que determinará la conveniencia de dicha prestación para cada caso particular. Es una decisión importante ya que se tienen que tener en cuenta no sólo los aspectos clínicos del paciente, sino los recursos con los que cuenta el centro de salud mental- infanto-juvenil (CSM-IJ) así como la capacidad de hacer psicoterapia del paciente y la adherencia de la familia al encuadre, entre otros aspectos.

La psicoterapia intensiva y con tiempos de aplicación largos, va ayudar a afrontar casos con problemas en diferentes áreas, ofreciendo habilidades para poder regular emociones, utilizando la relación terapéutica como escenario sobre el que sustentar la formación de relaciones interpersonales fructíferas y facilitando una cohesión y estructuración de la personalidad.

Se exponen a continuación algunos ejemplos de psicoterapia con evidencia empírica y clínica, que nos dan una visión de la necesidad de la intensidad y necesidad de tiempos largos. Los ejemplos de terapias que se exponen están centrados en patologías de personalidad y psicosis (que son trastornos que generan un gran gasto de recursos y una gran disfunción en el desarrollo de la persona) pero existen otras patologías y tratamientos con ejemplos de terapias largas e intensivas.

Para el caso de los trastornos de personalidad en la adolescencia, la terapia dialéctico conductual en su versión de adolescentes (Rathus y Miller, 2002) necesita una combinación de 16 semanas de tratamiento individual, más otros procedimientos terapéuticos para llegar al nivel 1 de tratamiento, pero el tiempo es de un año si se quiere llegar al nivel más alto propuesto por el programa de tratamiento (Linehan, 1993). Para tratamientos con problemas de personalidad y patología del yo, basados en las nuevas aproximaciones conductuales, se señala la necesidad de tratamientos psicoterapéuticos largos de un año o más (Kanter et al., 2010; Kohlenberg y Tsai, 1991).

En tratamientos de orientación psicoanalítica, también para trastornos de personalidad, se necesita un año o más, tanto para la terapia basada en la mentalización (Allen y Fonagy, 2008; Rossouw y Fonagy, 2012) como para la psicoterapia focalizada en la transferencia (Kernberg et al., 2008; Krischer y Normandin, 2015; Yeomans et al., 2016) así como para enfoques centrados en una visión psicodinámica e integradora (Foelsch et al., 2014) donde piden como mínimo seis meses con dos sesiones por semana. 
Desde una perspectiva cognitivo-conductual y para el tratamiento de psicosis en niños y adolescentes, se plantea un mínimo de 16 sesiones, para un tiempo mínimo entre 3 meses y un año de forma intensiva, en formato individual, acompañado de medicación y apoyo familiar (Gilbert et al., 2003; McGorry et al., 2008; NICE, 2016).

Los diferentes procesos de psicoterapia son distintos en su aplicación para las diferentes patologías. No obstante, todos ellos comparten una necesidad común, que es un marco de terapia largo e intensivo, sin el que no será posible el proceso de recuperación o mejora (ver tabla 1). Igualmente, todas ellas tienen como principal elemento la relación terapéutica y el establecimiento de una relación de calidad y fructífera.

Tabla 1. Claves descriptivas de Psicoterapias para TMG-IJ.

\begin{tabular}{|c|c|c|c|c|c|}
\hline & $\begin{array}{l}\text { Psicoterapia Focalizada } \\
\text { en la Transferencia } \\
\text { (Kernberg) }\end{array}$ & $\begin{array}{l}\text { Terapia Basada } \\
\text { en Mentalización } \\
\text { (Bateman y Fonagy }\end{array}$ & $\begin{array}{l}\text { Terapia Dialéctico } \\
\text { Conductual } \\
\text { (Linehan) }\end{array}$ & $\begin{array}{l}\text { Psicoterapia } \\
\text { Analítico Funcional } \\
\text { (Kholenberg y Tsai) }\end{array}$ & $\begin{array}{l}\text { TCC para psicosis } \\
\text { (basada en Beck) }\end{array}$ \\
\hline Principios & Psicoanalíticos & Psicoanalíticos & Conductuales & Conductuales & $\begin{array}{l}\text { Conductua- } \\
\text { les-Cognitivos }\end{array}$ \\
\hline Teoría & Difusión de identidad & Función reflexiva & $\begin{array}{l}\text { Modelo Biopsico- } \\
\text { social y dialéctica }\end{array}$ & $\begin{array}{l}\text { Historia de aprendizaje } \\
\text { Formación del YO }\end{array}$ & PI disfuncional \\
\hline $\begin{array}{l}\text { Mecanismos } \\
\text { de Cambio }\end{array}$ & Integración del Yo & Mentalización & $\begin{array}{l}\text { Aprendizaje y } \\
\text { reforzamiento }\end{array}$ & $\begin{array}{l}\text { Reforzamiento de } \\
\text { CCR2 y CCR3 }\end{array}$ & $\begin{array}{l}\text { Reforzamiento } \\
\text { de cogniciones } \\
\text { funcionales }\end{array}$ \\
\hline Expectativas & $\begin{array}{l}\text { Explicación racional sobre } \\
\text { aspectos de su personalidad } \\
\text { y relaciones de objeto }\end{array}$ & $\begin{array}{l}\text { Explicación racional } \\
\text { sobre personalidad } \\
\text { y aspectos del Yo }\end{array}$ & $\begin{array}{l}\text { Explicación racional } \\
\text { sobre su forma de } \\
\text { reaccionar por biografía }\end{array}$ & $\begin{array}{l}\text { Explicación relacional } \\
\text { y relacional sobre } \\
\text { historia biográfica }\end{array}$ & $\begin{array}{l}\text { Explicación sobre } \\
\text { formas de pensa- } \\
\text { miento en relación a } \\
\text { historia biográfica }\end{array}$ \\
\hline \multirow{5}{*}{$\begin{array}{l}\text { Acciones } \\
\text { terapéuticas }\end{array}$} & Contrato & Contrato & Contrato & Contrato & Contrato \\
\hline & Neutralidad & Interpretación & Jerarquía de objetivos & Evocar CCR & Reestructuración \\
\hline & Contratransferencia & & Aceptación & Emociones del terapeuta & \\
\hline & & & Activación & & \\
\hline & $\begin{array}{l}\text { Uso de la relación } \\
\text { terapéutica }\end{array}$ & $\begin{array}{l}\text { Uso de la relación } \\
\text { terapéutica }\end{array}$ & $\begin{array}{l}\text { Uso de la relación } \\
\text { terapéutica }\end{array}$ & $\begin{array}{l}\text { Uso de la relación } \\
\text { terapéutica }\end{array}$ & Alianza terapéutica \\
\hline \multirow[t]{3}{*}{ Encuadre } & Larga & Larga & 16 a 24 sesio- & Largas & \multirow{3}{*}{$\begin{array}{l}\text { Mínimo tres } \\
\text { meses a un año }\end{array}$} \\
\hline & $102 \mathrm{ss}$ & $102 \mathrm{ss}$ & & 102 sesiones & \\
\hline & 1 año & 1 año & + de año Pl & 10 más años & \\
\hline
\end{tabular}

Los procesos de cambio para trastornos graves necesitan intervenciones sostenidas en el tiempo y focalizadas en objetivos de trabajo. Los estudios señalan que para patologías menos graves las psicoterapias de tiempo corto y medio tienen su eficacia y muestran buenos resultados (Macdonald, 2011; Perkins y Scarlett, 2008), pero también se señala que las psicoterapias cortas no son suficientes para casos graves.

Para los trastornos de conducta resistentes, tratamientos de al menos un año, con una o dos sesiones a la semana, producen cambios significativos (Fonagy y Target, 1994). Y niños con sintomatología del vínculo internados en residencias, mejoran sus problemas si reciben sesiones de psicoterapia una vez a la semana durante dos años (Lush et al., 1991).

Para patologías graves, las intervenciones a corto plazo pueden tener un impacto negativo en los pacientes, bien porque no resuelven sus dificultades o bien porque generan sentimientos de fracaso en el paciente y los familiares, reduciendo las expectativas y el compromiso para posibles futuros tratamientos; además, se generan 
casos de "puerta giratoria" (Target, y Fonagy, 2002; Goodyer et al., 2011). Por otro lado, existe un proporción de pacientes con trastorno mental grave que van a presentar dificultades a largo plazo, por lo que necesitan intervenciones más intensivas y duraderas (Lasa-Zulueta et al., 2014; Meltzer, 2005; Mollejo Aparicio, 2012; Rutter y Smith, 1995).

Por lo tanto, es necesario poder distinguir entre aquellos pacientes que necesitan tratamientos cortos, que pueden llegar a ser el $25 \%$ según algunos estudios, de aquellos que necesitan tratamientos más intensivos, que van a necesitar más de 25 sesiones (Leichsenring y Rabung, 2011). No obstante para trastornos de personalidad se han realizado estudios en los que se ha observado que la mejoría se da cuando el intervalo de tratamiento está entre el año y los dos o tres años (Perry, Banon, y Ianni, 1999). Según la evidencia actual, los tratamientos estructurados e intensivos, desde diferentes enfoques teóricos, producen en estas patologías mejoras tanto a nivel ambulatorio como en hospitalización parcial (Bateman y Fonagy, 1999; Linehan et al., 2006; Miller et al., 2007).

Las experiencias sobre la aplicación de psicoterapias intensivas, estructuradas y largas, son escasas en nuestro entorno. No hay tan siquiera estudios sobre la psicoterapia en niños y adolescentes de forma global que permitan extraer conclusiones sobre la forma en que se está aplicando y con qué criterios de calidad. Existe una gran heterogeneidad en la aplicación de las mismas, así como aún falta un sistema de gestión que permita la correcta aplicación de dicha prestación, debido a las listas de espera, pocos programas específicos y distribución desigual de recursos (Cuellar et al., 2019; Asociación Madrileña de Salud Mental, 2015).

Hay pocas experiencias sanitarias de gestión que permitan la inclusión de psicoterapias intensivas largas, pero existen algunas estructuras sanitarias que propician espacios estructurados blindados para que se puedan realizar programas intensivos tanto psicoterapéuticos como psiquiátricos, como las experiencias de intervención compleja prolongada del Área de Gestión Clínica de Psiquiatría y Salud Mental del Hospital Universitario 12 Octubre (Jimenez Arriero, 2014), Servicio de Psiquiatría Infantil del Hospital Universitario Puerta de Hierro, PRISMA, (Hospital Universitario Gregorio Marañón), Fundación Orienta y Grupo TLP Barcelona, en el Hospital de Basurto.

La psicoterapia intensiva y con un tiempo adecuado, de no menos de un año, es imprescindible para la mejora de la población infanto-juvenil con trastornos mentales graves, y necesita de una formación profesional especializada, y con una certificación en psicoterapia para niños y adolescentes, principalmente en el campo de infancia y adolescencia, junto con un proceso de práctica clínica y feedback que permita una experiencia y pericia suficientes (Prado-Abril et al., 2017).

\section{Análisis económicos: otros modos de funcionamiento son posibles}

En este punto nos proponemos reflexionar sobre los aspectos económicos de la psicoterapia intensiva en niños con TMG-IJ. Para ello vamos a utilizar datos económicos publicados por la Comunidad de Madrid, y del precio de cada una de las prestaciones establecidas para los diagnósticos mentales. Hay que decir que los datos son generales y no están estratificados ni en diagnósticos infantiles al uso, ni en las prestaciones que se utilizan en el día a día.

Vamos a utilizar los grupos relacionados de diagnóstico (GRD) que son una herramienta de clasificación y gestión que permite relacionar diferentes pacientes que son atendidos en especializada con el coste que representa su asistencia siguiendo los criterios de gasto publicados por la Comunidad de Madrid (Consejería de Sanidad, 2017). En la tabla 2 se han elegido grupos diagnóstico relacionados con patologías de ingreso en unidades de hospitalización breve de adultos, de niños y adolescentes. El único GRD que existe para diagnósticos infantiles es de trastorno de comportamiento en la infancia.

Por lo que un GRD 754 (trastorno depresivo) con nivel 4 de gravedad equivale a un gasto de 13608 euros a nivel de atención especializada con ingresos hospitalarios incluidos. En el caso de trastornos de infancia el nivel de gasto por GRD 758 (trastornos del comportamiento en la infancia) con nivel 4 de gravedad es de 8071 euros, con ingreso hospitalario incluidos. A nivel de gasto en centros de salud ambulatorios, las prestaciones cobran otro carácter. Las primeras consultas, que suelen ser de una hora con independencia del trastorno, tienen un valor económico medio de 115 euros y las de seguimiento, que suelen ser de media hora o 45 minutos, 
Tabla 2. Gasto sanitario por GRD en Salud Mental con niveles de gravedad

\begin{tabular}{|c|c|c|c|c|c|c|}
\hline & & GRD & & Nivel d & ravedad & \\
\hline Diagnóstico & \multirow{6}{*}{$\begin{array}{l}\text { क } \\
\frac{0}{\bar{Z}} \\
\frac{0}{q}\end{array}$} & & 1 & 2 & 3 & 4 \\
\hline Esquizofrenia & & 750 & $7.142 €$ & $7.905 €$ & $11.426 €$ & $13.139 €$ \\
\hline $\begin{array}{l}\text { Trastornos depresivos mayores y otros/psicosis no espe- } \\
\text { cificadas }\end{array}$ & & 751 & $7.384 €$ & $8.188 €$ & $9.126 €$ & $10.537 €$ \\
\hline Trastornos de personalidad y control de impulsos & & 752 & $5.180 €$ & $5.475 €$ & $6.569 €$ & $6.569 €$ \\
\hline Depresión excepto trastorno depresivo mayor & & 754 & $3.096 €$ & $4.124 €$ & $6.162 €$ & $13.608 €$ \\
\hline Trastornos de adaptación y neurosis & & 755 & $3.356 €$ & $5.334 €$ & $8.175 €$ & $12.336 €$ \\
\hline Trastorno de comportamiento en la infancia. & $\stackrel{\substack{C \\
\sum}}{2}$ & 758 & $3.303 €$ & $4.235 €$ & $6.107 €$ & $8.071 €$ \\
\hline
\end{tabular}

Nivel de gasto por proceso asistencia de hospitalización en Euros, por grupo diagnóstico según BOCAM (2017).

de 70 euros. Por lo tanto, los precios establecidos en el caso de hospitalización y especializada son diferentes que los establecidos en los centros de salud mental ambulatoria.

Con estos precios vamos a realizar una estimación de lo que podría suponer el gasto sanitario de un proceso de psicoterapia intensiva, para un trastorno de comportamiento en la infancia (GRD 758). No sabemos si las autoridades consideran que dentro de esta categoría es igual un trastorno de ansiedad o autismo, o psicótico. No obstante, da la impresión de que el tener un trastorno de comportamiento en la infancia es menos costoso (salvo si se compara con los trastornos de personalidad) que otros GRD.

En el supuesto de un paciente, con impresión diagnóstica de trastorno de comportamiento en la infancia (GRD 758), que acude a consulta de especialista en psiquiatría de la infancia y adolescencia o en psicología clínica de la infancia y adolescencia, y se decidiera realizar una evaluación minuciosa, dedicando 5 sesiones que incluyan entrevistas familiares, de diagnóstico y administración de pruebas psicodiagnósticas, podríamos establecer para el cálculo de gasto 5 entrevistas de una hora de duración, considerándolas como nuevas, según los criterios de atención ambulatoria descritos arriba. Por lo que el gasto sanitario sería de 575 euros, para 5 consultas de diagnóstico en un caso de trastorno de comportamiento en la infancia. Podría ser que este GRD genérico, pueda incluir un trastorno depresivo grave o un primer brote psicótico en la adolescencia o un trastorno de personalidad en la adolescencia. Por lo que tendríamos un TMG-IJ, bien diagnosticado con 5 sesiones, con un precio de 575 euros.

Tras este proceso de diagnóstico, el paciente podría entrar en la Comisión TMG-IJ, y entre los profesionales decidir las propuestas terapéuticas. Que incluirían, además del tratamiento farmacológico pautado, y las entrevistas a nivel de trabajo social o de enfermería necesarias, (cuyos gastos habría que calcular ${ }^{1}$ ), si se decide emplear con dicho paciente una psicoterapia intensiva de una duración determinada.

\footnotetext{
${ }^{1}$ Ni el BOCAM del 2017, ni en otros documentos oficiales a nivel público aparecen gastos desglosados a nivel de intervenciones específicas ni de roles profesionales, así como tampoco existe un desglose por diferentes cuadros psicopatológicos de la infancia y adolescencia.
} 
Si se decide prescribir al paciente la prestación de psicoterapia intensiva, con una duración de un año (48 sesiones) a año y medio (72 sesiones), el coste de la intervención sería de 3935 a 5615 euros en total (ver tabla 3). En ningún caso ascendería al gasto por GRD al nivel cuatro de trastornos de comportamiento en la infancia con ingreso hospitalario que sería de 8071 euros, por lo que el ahorro sería alto, (sin contar los seguimientos farmacológicos, más las intervenciones a nivel social y de enfermería).

Es decir, que la estimación realizada, señala que los gastos por una psicoterapia intensiva para un caso de trastorno del comportamiento grave (5 615 euros), son menores que los gastos que implicaría el ingreso de un niño en UHB por la misma problemática (8 071 euros).

Tabla 3. Gastos por procesos de terapia

\begin{tabular}{llcccc}
\hline Niveles & Trastorno General & Diagnóstico & Sesiones & Psicoterapia & Total \\
\hline $3=12$ Meses & $\begin{array}{l}\text { Trastornos del comportamien } \\
\text { to en la infancia (GRD 758) }\end{array}$ & $5 \times 115=575 €$ & 48 & $48 \times 70=3.360 €$ & $3935 €$ \\
\hline $4=18$ Meses & (GRD 758) & $5 \times 115=575 €$ & 72 & $72 \times 70=5.040 €$ & $5615 €$ \\
\hline
\end{tabular}

Los datos ofrecidos son una estimación de lo que podría ser un proceso efectivo, justo y asociado al encuadre que ofrece garantías de eficacia, eficiencia y efectividad.

En resumen, lo que queremos destacar con esta estimación de datos económicos, es que una intervención prolongada semanal, para niños con TMG-IJ, es factible y no supone tanto gasto como lo que supone no intervenir de manera adecuada, dando lugar a ingresos que son más caros. El ahorro es considerable.

Ahora vamos a presentar algunos datos que señalan la pertinencia de organizar y tratar a los niños y adolescentes con trastorno mental grave de una forma intensiva.

\section{Psicoterapia para TMG-IJ en otros sistemas de salud}

En Reino Unido se estudió el gasto invertido en relación al beneficio económico encontrado, se recoge que para trastorno de ansiedad en la infancia, por cada libra que el sistema invierte en gasto, existe un beneficio de 31 libras en los costes indirectos que supondría no hacer la inversión; en trastornos depresivos, si se usa la terapia cognitivo-conductual para niños, el beneficio es de 31 libras por cada libra gastada, y en el caso de intervención con padres, por cada libra gastada existe un beneficio de 10 libras (Khan, Parsonage, y Stubbs, 2015).

Cada libra invertida en la terapia grupal cognitivo-conductual para la ansiedad en la adolescencia produce beneficios de casi 7 libras (WSIPP, 2018). Los programas de terapia parental grupal para trastornos de conducta en niños pequeños son eficaces, y pueden generar beneficios de al menos 3 libras por cada libra invertida (Parsonage et al., 2014).

De estos aspectos se hacen eco el sistema de salud de Reino Unido, que pone en marcha el Children and Young People's Improving Acces To Psychological Programe [mejora del acceso a programas psicológicos para niños y jóvenes] (https://cypiapt.com), donde se establecen estrategias a nivel nacional de los tratamientos psicológicos en los centros de salud mental, con el fin de aumentar la implementación de tratamientos psicoterapéuticos en niños y adolescentes, así como la evaluación de tratamientos basados en la evidencia para trastornos comunes y graves, llevando a cabo entrenamientos a los profesionales que prestan servicios de salud mental (National Health Service (NHS), 2015; Peter Fonagy et al., 2017). 
En otros países del entorno europeo, se llevan a cabo prácticas diferentes en cuanto a la aplicación de las psicoterapias, teniendo presente la importancia de generar estructuras sanitarias propicias al desarrollo de psicoterapias intensivas.

El modelo inglés, con los diferentes niveles de atención a la salud mental de niños y adolescentes, plantea en el nivel 3 y 4, para trastornos graves (p. ej: trastornos psicóticos, trastorno de personalidad, trastornos afectivos....), la aplicación de tratamientos basados en la evidencia, donde a nivel de salud mental especializada, equivalente en nuestro país a atención ambulatoria y de hospitalización parcial, cubre la prestación de psicoterapia con un mínimo de 14 sesiones, que se pueden ir ampliando con paquetes de 25 sesiones, y que se va alargando hasta que se necesite (Wolpert et al., 2014.; Wolpert et al., 2016). Este modelo ha estado influido por la creación de una Comisión para el Avance de Tratamientos Psicológicos en niños y adolescentes - CYPIAPT dentro del Servicio Nacional de Salud de Reino Unido (National Health System, s. f. ) , así como una Comisión de Estudio de Resultados de Coste Beneficio de los Tratamientos aplicados en Salud Mental Infanto-juvenil.

El sistema sanitario alemán brinda una amplia cobertura a la salud mental infanto-juvenil. Una comisión de evaluación diagnóstica valora si el paciente tiene un TMG-IJ, y se prescriben o terapia breve (Kurzzeittherapie), consistente en 25 horas a razón de 50 minutos por sesión, o terapia prolongada (Langzeittherapie), que dependiendo de cuál sea método o escuelas terapéuticas así se estiman las horas de tratamiento. En cuanto a esto, en Alemania están reconocidas: la terapia cognitivo-conductual, la terapia analítica (psicoanálisis) y la terapia fundada en la psicología profunda (psicodinámica). Si el caso es de mayor gravedad psicopatológica, más derecho tiene el niño o adolescente a recibir psicoterapia más prolongada e intensiva. La terapia analítica se distribuye en 70 horas, a razón de 3 horas semanales, que se conceden en una primera solicitud por parte del psicoterapeuta y después se puede prolongar 150 horas más en el caso de la psicoterapia de niños y 180 horas más en el de adolescentes. En la práctica supone que los menores con psicopatología grave pueden recibir tratamiento psicoterapéutico durante 3 años completamente costeado por el sistema sanitario alemán. Para la terapia fundada en la psicología profunda son 70 horas para niños y 90 horas para adolescentes que pueden prologarse 150 horas y 180 horas más respectivamente. Con este método psicoterapéutico suele trabajarse a razón de una (en algunos casos dos) horas a la semana. Asimismo, para la terapia de conducta que suele aplicarse una vez a la semana se conceden inicialmente 60 horas de tratamiento que pueden prolongarse otras 80 más. Esto significa que los niños y adolescentes en Alemania tienen el derecho a recibir la psicoterapia que precisen dependiendo de la indicación y gravedad del trastorno psicopatológico con una frecuencia semanal de entre 1 y 3 sesiones semanales y durante varios años (Bundes Psychotherapeuten Kammer, 2018).

En Finlandia, también existe la posibilidad de psicoterapias intensivas desde 80 a 225 sesiones para edades de entre 16 y 25 años (Kela, 2017). En Estocolmo, el proyecto STOPP financiado por el gobierno, estudia la efectividad de las psicoterapias psicodinámicas intensivas de más de un año de un duración (Sandell, 1999; Sandell et al., 2000).

Se aprecia por tanto que otros sistemas de salud, con sus modelos propios de gestión, no solo hacen análisis económicos de las diferentes alternativas terapéuticas, sino que tienen claro que, a mayor grado de psicopatología, mas compromiso se tiene que dar en la salud mental infanto-juvenil, y no dudan en ofrecer tratamientos largos ambulatorios.

\section{Conclusiones}

Hemos querido en este trabajo exponer la importancia de la psicoterapia intensiva ambulatoria en el trabajo con niños y adolescentes, y sobre todo en el trabajo con patologías graves. En nuestro país no existe por ahora una estructura de gestión ni una conciencia real sobre dicha prestación, ya que no existen datos claros sobre la forma en que se aplica, a quién va dirigida, y por supuesto no existen análisis económicos sobre su aplicación y los efectos de la misma, ni para trastornos moderados ni graves de la infancia y adolescencia, sí en adultos (Ruiz-Rodríguez et al., 2017). Tampoco existe un análisis sobre el impacto que tienen los trastornos mentales graves en la infancia y adolescencia en las familias, en el colegio, en el sistema judicial ni en el propio sistema de salud. 
Es necesario, en primer lugar, un ajuste de los métodos de categorización de diagnósticos en la salud mental infanto-juvenil a las políticas de gestión, que están principalmente asociadas a criterios diagnósticos de adultos. Sería necesario también un desglose de los gastos económicos que suponen a nivel de salud mental ambulatoria los diferentes profesionales, como análisis de la actividad realizada y los resultados de las intervenciones. Datos desglosados, con facilidad de acceso por parte de los profesionales, con el fin de reflexionar sobre las propias prácticas de una forma concienzuda.

Los problemas mentales en la infancia y adolescencia, tanto los leves y moderados como graves, son numerosos y no siempre reciben la atención adecuada. Sirva como ejemplo que, a día de hoy, la mayoría de los trastornos del espectro autista no pueden ser asumidos por el sistema sanitario y son las organizaciones de familiares y el asociacionismo el que responde a sus demandas de tratamiento.

Por lo tanto, es necesaria la inclusión en los centros de salud mental ambulatorios infanto-juveniles de programas dedicados exclusivamente al TMG-IJ, con espacios en la agenda, con reuniones marcadas entre los diferentes profesionales y con recursos humanos que permitan incluir la prestación de psicoterapia intensiva ambulatoria dentro de los itinerarios terapéuticos.

Una gestión centrada en la discriminación en las primeras consultas entre patologías leves, moderadas y graves al modo del modelo inglés Thrive (Wolpert et al., 2016) podría dar respuesta a la alta demanda de consultas de salud mental, así como permitiría establecer procesos de gestión específicos para el TMG-IJ.

El proceso de gestión de TMG-IJ en las unidades de salud mental, implicará una mayor coordinación con las unidades de atención colindantes (pediatría, atención primaria, hospital de día, ...) y tendría que ser una prioridad en el próximo Plan de Salud Mental nacional, donde se marcaran las líneas de creación de dichos programas y/o unidades de TMG-IJ, así como la necesaria dotación de recursos.

La psicoterapia intensiva ambulatoria es una prestación que, debido a las características del sistema sanitario, y dentro de un contexto público, consideramos imprescindible sea llevada a cabo por un psiquiatra especialista en infancia y adolescencia o un especialista en psicología clínica en infancia y adolescencia. Modalidad terapéutica que sin duda tendría que contemplarse en los programas de formación de las futuras especialidades.

Creemos que esto es posible, porque en otros países está siendo posible, y porque se ha demostrado que a largo plazo ahorra dinero. Permite un crecimiento del niño y del adolescente, no solo controlando sus síntomas, sino generando un fortalecimiento de sus recursos y personalidad, que redundaría en una reducción del uso de la medicalización y hospitalización, a la vez que favorecería el establecimiento del vínculo con un psicoterapeuta en el centro de salud mental que puede constituir un lugar seguro para resolver crisis futuras.

El mapa que existe de la atención al TMG-IJ en el Estado español es heterogéneo, y muy débil aún. Este trabajo pretende poner de relieve dichas deficiencias, señalando que es necesario ya un análisis no sólo del impacto económico de los trastornos mentales en la infancia y adolescencia, sino que es necesario estudiar qué prestaciones son las más adecuadas, y dentro de ellas cómo la psicoterapia puede dar respuesta.

La psicoterapia intensiva ambulatoria es una cuestión necesaria y de derechos humanos en el niño que debemos reclamar y que no podemos subestimar ni dejar de lado en nuestro trabajo diario. Creemos que la psicoterapia intensiva ambulatoria debe ofrecerse como ya se hace en otros países señalados, y que tiene que ser en los centros de salud mental ambulatarios infanto-juveniles, siempre y cuando se dote de un mayor número de recursos.

Es imprescindible la inclusión de una mayor atención de calidad al TMG-IJ en la red, aprovechando los recursos de formación sanitaria especializada que ofrece la formación en psiquiatría y psicología clínica, con sus futuras especialidades, así como un fortalecimiento de estos contenidos en los programas de docencia en las unidades multiprofesionales. Potenciando el papel de la atención por parte de enfermería de salud mental y de trabajo social.

La psicoterapia intensiva ambulatoria es una cuestión de derechos humanos y de los derechos del niño y este trabajo intenta poner de relieve la necesidad de hacer las cosas mejor al respecto. 


\section{Referencias}

Aláez Fernández, M., Martínez-Arias, R. y Rodríguez-Sutil, C. (2000). Prevalencia de trastornos psicológicos en ninos y adolescentes, su relación con la edad y el género. Psicothema, 12(4), 525-532.

Allen, J. G. y Fonagy, P. (2008). Handbook of Mentalization-Based Treatment. Handbook of Mentalization-Based Treatment. John Wiley \& Sons. https://doi.org/10.1002/9780470712986

Arnett, J. J. (Ed.). (2007). From Emerging Adulthood to Young Adulthood. En Autor, Emerging Adulthood: The Winding Road from the Late Teens through the Twenties (pp. 207-228). Oxford Scholarship Online. https://doi.org/10.1093/acprof:oso/9780195309379.003.0010

Association of Child Psychotherapists. (s. f.). Child and adolescent psychotherapy: longer-term and more intensive work. Briefing Papers Series. https://childpsychotherapy.org.uk/sites/default/files/documents/CAPTlonger-term-and-more-intensive-work.pdf

Bardón, C. (2012). Los trastornos mentales graves en la infancia y la adolescencia. L'Interrogant, 6. https://revistainterrogant.org/los-trastornos-mentales-graves-la-infancia-la-adolescencia/

Basterra, V.(2016). Porcentaje de población infanto-juvenil española con problemas psicoemocionales y sus diferencias entre 2006 y 2012. Medicina Clínica, 147, 393-396. https://doi.org/10.1016/j.medcli.2016.07.019

Bateman, A., y Fonagy, P. (1999). Effectiveness of partial hospitalization in the treatment of borderline personality disorder: A randomized controlled trial. American Journal of Psychiatry, 156(10), 1563-1569. https://doi.org/10.1176/ajp.156.10.1563

Beecham, J. (2014). Annual research review: Child and adolescent mental health interventions: A review of progress in economic studies across different disorders. Journal of Child Psychology and Psychiatry and Allied Disciplines, 55(6), 714-732. https://doi.org/10.1111/jcpp.12216

Blomberg, J., Lazar, A. y Sandell, R. (2010). Long-Term Outcome of Long-Term Psychoanalytically Oriented Therapies: First Findings of the Stockholm Outcome of Psychotherapy and Psychoanalysis Study. Psychotherapy Research, 11(4), 361-382. https://doi.org/10.1093/ptr/11.4.361"10.1093/ptr/11.4.361

Bragado, C., Bersabé, R. y Carrasco, I. (1999). Factores De Riesgo Para Los En Niños Y Adolescentes. Psicothema, 11(4), 939-956.

Bundes Psychotherapeuten Kammer (BPtK). (2018). Paths to Psychotherapy. https://www.bptk.de/wp-content/uploads/2019/09/2019-09 bptk patientenbroschuere englisch web.pdf

Care Quality Comission (2017). Review of children and young people's mental health services. https://www. cqc.org.uk/sites/default/files/20171103 cypmhphase1 report.pdf

Consejería de Sanidad. (2017). Orden 727/2017, de 7 de agosto, del Consejero de Sanidad, por la que se fijan los precios públicos por la prestación de los servicios y actividades de naturaleza sanitaria de la red de centros de la Comunidad de Madrid.

Consejo General de la Psicología de España. (14 de marzo, 2019). Es fundamental mejorar la atención y promoción de la salud y bienestar emocional de los jóvenes, según una PNL. Infocop online. http://www. infocop.es/view article.asp?id $=8009 \&$ cat $=50$

Cuellar, I., Izquierdo, A. y Padilla, D. (2019). Mapa sobre la Psicología Clínica Infanto-Juvenil. ¿Una nueva especialidad sanitaria?. Clínica Contemporánea, 10(2), Artículo e13. https://doi.org/10.5093/cc2019a10

Dirección General de la Familia y el Menor. (Coord.). (2017). Plan de Infancia y Adolescencia de la Comunidad de Madrid (2017-2021). https://www.comunidad.madrid/transparencia/sites/default/files/plan/document/plan de infancia y adolescencia publicamadrid.pdf

Faulconbridge, J., Law, D. y Laffan, A. (2015). What good looks like in psychological services for children, young people and their families. The Child \& Clinical Psychology Review, 3. https://www.bps.org. uk/sites/bps.org.uk/files/Blogs/Files/The\%20Child\%20\%26\%20Family\%20Clinical\%20Psychology\%20 Review\%20-\%20Summer\%202015.pdf

FEAP. (2007). Documento base de psicoterapia en el Sistema Nacional de Salud. http://www.feap.es/images/ feap/documentos/psicoterapia en el sistema nacional de salud.pdf

Fernández Liria, A. y Gómez Beneito, M. (Coords.). (2009). Informe sobre la salud mental de niños y adolescentes. Asociación Española de Neuropsiquiatría. https://consaludmental.org/centro-documentacion/informe-salud-mental-ninos-adolescentes/ 
Foelsch, P. A., Schlüter-Müller, S., Odom, A. E., Arena, H. T., Borzutzky H., A. y Schmeck, K. (2014). Adolescent Identity Treatment. An Integrative Approach for Personality Pathology. New York: Springer.

Fonagy, P., Pugh, K. y O'Herlihy, A. (2017). The Children and Young People's Improving Access to Psychological Therapies (CYP IAPT) Programme in England. En D. Skuse, H. Bruce y L. Dowdney (Eds.), Child Psychology and Psychiatry (cap. 48, pp. 429-435). John Wiley\&Sons. https://doi.org/10.1002/9781119170235. $\underline{\mathrm{ch} 48}$

Fonagy, P. y Target, M. (1994). The efficacy of psychoanalysis for children with disruptive disorders. Journal of the American Academy of Child and Adolescent Psychiatry, 33(1), 45-55. https://doi.org/10.1097/00004583199401000-00007

Gilbert, M., Miller, K., Berk, L., Ho, V. y Castle, D. (2003). Scope for psychosocial treatments in psychosis: An overview of collaborative therapy. Australasian Psychiatry, 11(2), 220-224. https://doi.org/10.1046/1.10398562.2003.00555.x

Green, V. (2009). Individual psychotherapy: assessment, intensive and non-intensive work. En M. Lanyado y A. Horne (Eds.), The Handbook of Child and Adolescent Psychotherapy (pp. 175-191). Routledge.

Jiménez Arriero, M. A. (2014). Memoria del Área de Gestión Clínica de Psiquiatría y Salud Mental Hospital Universitario 12 Octubre. Consejería de Sanidad de la Comunidad Autónoma de Madrid.

Jones, P. J., Mair, P., Kuppens, S. y Weisz, J. R. (2019). An Upper Limit to Youth Psychotherapy Benefit? A Meta-Analytic Copula Approach to Psychotherapy Outcomes. Clinical Psychological Science, 7(6), 14341449. https://doi.org/10.1177/2167702619858424

Junta Asociación Madrileña de Salud Mental. (2015). Informe Sobre Evolución De Recursos De Salud Mental De La Comunidad De Madrid 2000-2015. Asociación Española de Neuropsiquiatría. https://amsmblog.files. wordpress.com/2016/05/evoluciocc81n-de-recursos-de-salud-mental-2000-2015.pdf

Kanter, J. W., Tsai, M. y Kohlenberg, R. J. (2010). The Practice of Functional Analytic Psychotherapy. Springer. https://doi.org/10.1007/978-1-4419-5830-3

Kela. (5 de junio de 2017). Rehabilitative psychotherapy. https://www.kela.fi/web/en/rehabilitative-psychotherapy

Kernberg, O. F., Krischer, M. K. y Foelsch, P. A. (2008). Transference focused psychotherapy for adolescents: a preliminary communication. Praxis Der Kinderpsychologie Und Kinderpsychiatrie, 57(8-9), 662-692.

Kessler, R. C., Berglund, P., Demler, O., Jin, R., Merikangas, K. R. y Walters, E. E. (2005). Kessler RC, Berglund P, Demler O, Jin R, Walters EE. Lifetime prevalence and age-ofonset distributions of DSMIV disorders in the National Comorbidity Survey Replication. Archives of General Psychiatry, 62(6), 593-602.

Khan, L., Parsonage, M. y Stubbs, J. (2015). Investing in children's mental health: a review of evidence on the costs and benefits of increased service provision. Centre for Mental Health. https://www.centreformentalhealth.org.uk/Handlers/Download.ashx?IDMF=f67f310a-e9b4-4cef-be9f-7f985f21dcea

Knapp, M., Ardino, V., Brimblecombe, N., Evans-Lacko, S., Iemmi, V., King, D., Snell, T., Murguia Asensio, S., Mbeah-Bankas, H., Crane, S., Harris, A., Fowler, D., Hodgekins, J. y Wilson, J. (2016). Youth Mental Health: New Economic Evidence. Personal Social Services Research Unit (PSSRU). https://www.pssru. ac.uk/publications/pub-5160/

Kohlenberg, R. J. y Tsai, M. (1991). Functional analytic psychotherapy: Creating intense and curative therapeutic relationschips. Plenum.

Krischer, M. K., y Normandin, L. (2015). Tratamiento de adolescentes con trastorno límite en un hospital de día mediante técnicas de la psicoterapia focalizada en la transferencia (TFP). Revista de Psicopatología y Salud Mental del Niño y del Adolescente 26, 49-59.

Laffan, A., Faulconbridge, J., Hunt, K. y O'Curry, S. (2016). Faculty for Children, Young People and their Families - We believe the children are our future: Reflections and aspirations from the Faculty for Children, Young People and their Families. Clinical Psychology Forum, 277, 13-17.

Lasa-Zulueta, A., Jorquera-Cuevas, C., Solana-Azurmendi, B. y Del Arco-Heras, S. (2014). Evaluación de la calidad asistencial en el SNS de los trastornos mentales graves en la infancia. Estudio en salud mental infanto-juvenil. Servicio Central de Publicaciones del Gobierno Vasco. 
Leichsenring, F. y Rabung, S. (2011). Long-term psychodynamic psychotherapy in complex mental disorders: Update of a meta-analysis. British Journal of Psychiatry, 199(1), 15-22. https://doi.org/10.1192/bjp. bp.110.082776

Linehan, M. (1990). Cognitive-behavioral treatment of borderline personality disorder. The Guilford.

Linehan, M. M., Comtois, K. A., Murray, A. M., Brown, M. Z., Gallop, R. J., Heard, H. L., ... Lindenboim, N. (2006). Two-year randomized controlled trial and follow-up of dialectical behavior therapy vs therapy by experts for suicidal behaviors and borderline personality disorder. Archives of General Psychiatry, 63(7), 757-766. https://doi.org/10.1001/archpsyc.63.7.757

Lush, D., Boston, M., y Grainger, E. (1991). Evaluation of psychoanalytic psychotherapy with children: Therapists' assessments and predictions. Psychoanalytic Psychotherapy, 5(3), 191-234. https://doi. org/10.1080/02668739100700101

Macdonald, A. (2011). Solution-focused therapy: Theory, research and practice. Sage Publications.

McGorry, P. D., Killackey, E. y Yung, A. (2008). Early intervention in psychosis: concepts, evidence and future directions. World Psychiatry, 7(3), 148-156. https://doi.org/10.1002/j.2051-5545.2008.tb00182.x

McGorry, P. D., Purcell, R., Hickie, I. B. y Jorm, A. F. (2007). Investing in youth mental health is a best buy. Medical Journal of Australia, 187(S7). https://doi.org/10.5694/j.1326-5377.2007.tb01326.x

Meltzer, H. (2005). The nature and scale of the problem - The prevalence of mental health problems and mental disorders in childhood and adolescence. En R. Williams y M. Kerfoot (Eds.), Child and adolescent mental health services, strategy, planning delive. Oxford University Press.

Midgley, N., Capella, C., Goodman, G., Lis, A., Noom, M., Tishby, O., y Weitkamp, K. (2018). Introduction to the special section on child and adolescent psychotherapy research. Psychotherapy Research, 28(1), 1-2. https://doi.org/10.1080/10503307.2017.1380864

Miller, A.L; Rathus, J.H y Linehan, M. (2007). Dialectical Behavior Therapy with Suicidial Adolescents. Guilford.

Miller, S. D., Hubble, M. A., Chow, D. L., y Seidel, J. A. (2013). The outcome of psychotherapy: Yesterday, today, and tomorrow. Psychotherapy, 50(1), 88-97. https://doi.org/10.1037/a0031097

Mollejo Aparicio, E. (2012). Los trastornos mentales graves en la infancia y la adolescencia. Asociación Española de Neuropsiquiatría.

National Health System. (s. f.). Adult Improving Access to Psychological Therapies programme. https://www. england.nhs.uk/mental-health/cyp/iapt/

Navarro-Gómez, N. (2017). El suicidio en jóvenes en España: cifras y posibles causas. Análisis de los últimos datos disponibles. Clínica y Salud, 15, 1-97. Retrieved from http://www.redalyc.org/ pdf/1806/180617820005.pdf

NICE. (2016). Psychosis and schizophrenia in children and young people: recognition and management. National Institute for Health and Care Excellence. https://www.nice.org.uk/guidance/cg 155

O’Brien, J., Christner, J., Biermann, B., Felt, B., Van Harrison, R., Kochhar, P. y Streetman, D.-A. (2013). Attention-deficit Hyperactivity Disorder. University of Michigan Health Service.

Observatorio de Infancia. (2013). II Plan Estratégico Nacional de Infancia y Adolescencia 2013-2016. Ministerio de Sanidad, Servicios Sociales e Igualdad. https://observatoriodelainfancia.vpsocial.gob.es/documentos/pdf/II PLAN ESTRATEGICO INFANCIA.pdf

Oficina Regional de Coordinación de Salud Mental. (Coord,). (2018). Plan Estratégico de Salud Mental de la Comunidad de Madrid 2018-2020. Dirección General de Coordinación de la Asistencia Sanitaria. Servicio Madrileño de Salud. http://www.madrid.org/bvirtual/BVCM020214.pdf

Organización Mundial de la Salud. (2018). Adolescent Health. https://www.who.int/health-topics/adolescent-health\#tab=tab 1

Patton, G. C., Olsson, C. A., Shirbekk, V., Saffert, R., Wlodek, M. E., Azzopardi, P. S., Stonawski, M., Rasmussen, B., Spry, E., Francis, K., Bhutta, Z. A., Kassebaum, N. J., Mokdad, Al. H., Murray. C. J. L., Prentice, A. M., Reavley, N., Sheehan, P., Sweeny, K., Viner, R. M. y Sawyer, S. M. (2018). Adolescence and the next generation. Nature, 554(7693), 458-466. https://doi.org/10.1038/nature25759 
Paus, T., Keshavan, M. y Giedd, J. N. (2008). Why do many psychiatric disorders emerge during adolescence? Nature Reviews Neuroscience. https://doi.org/10.1038/nrn2513

Pérez-álvarez, M. (2019). La psicoterapia como ciencia humana, más que tecnológica. Papeles del Psicólogo, 40(1), 1-17.

Pérez Álvarez, M. (2010). Guía de tratamientos psicológicos eficaces (T. III): Infancia y adolescencia. Pirámide.

Perkins, R. y Scarlett, G. (2008). The effectiveness of single session therapy in child and adolescent mental health. Part 2: An 18-month follow-up study. Psychology and Psychotherapy: Theory, Research and Practice, 81(2), 143-156. https://doi.org/10.1348/147608308X280995

Perry, J. C., Banon, E. y Ianni, F. (1999). Effectiveness of psychotherapy for personality disorders. American Journal of Psychiatry, 156(9), 1312-1321. https://doi.org/10.1176/ajp.156.9.1312

Prado-Abril, J., Sánchez-Reales, S. e Inchaustic, F. (2017). En busca de nuestra mejor versión: pericia y excelencia en Psicología Clínica. Ansiedad y Estrés, 23(2-3), 110-117. https://doi.org/ 10.1016/j.anyes.2017.06.001

Rathus, J. H., y Miller, A. L. (2002). Dialectical behavior therapy adapted for suicidal adolescents. uicide and Life Threatening Behaviour, 32(2),146-57. htpps://doi.org/ 10.1521/suli.32.2.146.24399

Rossouw, T. I., y Fonagy, P. (2012). Mentalization-based treatment for self-harm in adolescents: A randomized controlled trial. Journal of the American Academy of Child and Adolescent Psychiatry, 51(12). https://doi. org/10.1016/j.jaac.2012.09.018

Ruiz-Rodríguez, P., Cano-Vindel, A., Muñoz Navarro, R., Medrano, L., Moriana, J. A., Buiza Aguado, C., Jiménez Cabré, G., González-Blanch, C. y Grupo de Investigación de PsicAP. (2017). Impacto económico y carga de los trastornos mentales comunes en España: una revisión sistemática y crítica. Ansiedad y Estrés, 23(2-3), 118-123. https://doi.org/10.1016/j.anyes.2017.10.003

Rutter, M y Smith, D. J. (1995). Psychosocial disorders in young people: The trends and their causes. Wiley.

Sandell, R., Blomberg, J., Lazar, A., Carlsson, J., Broberg, J., y Schubert, J. (2000). Varieties of long-term outcome among patients in psychoanalysis and long-term psychotherapy: A review of findings in the stockholm outcome of psychoanalysis and psychotherapy project (STOPP). International Journal of Psycho-Analysis, 81(5), 921-942. https://doi.org/10.1516/0020757001600291

Scott, S., Knapp, M., Henderson, J., y Maughan, B. (2002). Financial cost of social exclusion: follow up study of antisocial children into. The British Medical Journal, 323(7306), 191-191. https://doi.org/10.1136/ bmj.323.7306.191

Shedler, J. (2010). The Efficacy of Psychodynamic Psychotherapy. American Psychologist, 65(2), 98-109. https://doi.org/10.1037/a0018378

Shirk, S. (Ed.). (1988). Casual reasoning and children comprehension of terapeutic interpretions. En Cognitive Development and Child Psychotherapy. Perspectives in Developmental Psychology (pp. 53-89). Springer. https://doi.org/10.1007/978-1-4899-3635-6 3

Shirk, S. R., Karver, M. S., y Brown, R. (2011). The Alliance in Child and Adolescent Psychotherapy. Psychotherapy, 48(1), 17-24. https://doi.org/10.1037/a0022181

Simonoff, E., Pickles, A., Charman, T., Chandler, S., Loucas, T., y Baird, G. (2008). Psychiatric disorders in children with autism spectrum disorders: Prevalence, comorbidity, and associated factors in a population-derived sample. Journal of the American Academy of Child and Adolescent Psychiatry, 47(8), 921-929. https:// doi.org/10.1097/CHI.0b013e318179964f

Skokauskas, N., Lavelle, T. A., Munir, K., Sampaio, F., Nystrand, C., McCrone, P., McDaid, D., Chisholm, D., Byford, S., Ganguli, P., Feldman, I. y Belfer, M. (2018). The cost of child and adolescent mental health services. The Lancet Psychiatry, 5(4), 299-300. https://doi.org/10.1016/s2215-0366(18)30089-0

Suhrcke, M., Pillas, D. y Selai, C. (2008). Economic aspects of mental health in children and adolescents. In Social Cohesion for Mental Wellbeing among adolescents (pp. 43-64). WHO.

Target, M y y Fonagy, P. (2002). Anna Freud Centre studies 3: The long-term follow- up of child analytic treatments (AFC3). En P. Fonagy (Ed.). An open door review of outcome studies in psychoanalysis (pp. 206214). International Psychoanalytical Association. https://doi.org/10.13140/2.1.3458.0160 
Trowell, J., Joffe, I., Campbell, J., Clemente, C., Almqvist, F., Soininen, M., Koskenranta-Aalto, U., Weintraub, S., Kolaitis, G., Tomaras, V., Anastasopoulus, D., Grayson, K., Barnes, J. y Tsiantis, J. (2007). Childhood depression: A place for psychotherapy - An outcome study comparing individual psychodynamic psychotherapy and family therapy. European Child and Adolescent Psychiatry, 16(3), 157-167. https://doi. org/10.1007/s00787-006-0584-X

Volkmar, F. R., Reichow, B. y McPartland, J. C. (Eds.). (2014). Adolescents and adults with autism spectrum disorders. Springer. https://doi.org/10.1007/978-1-4939-0506-5

Wampold, B. E. y Imel, Z. E. (2015). The great psychotherapy debate: The evidence for what makes psychotherapy work: Second edition. The Great Psychotherapy Debate: The Evidence for What Makes Psychotherapy Work (2a edición). https://doi.org/10.4324/9780203582015

Wolpert, M., Harris, R., Hodges, S., Fuggle, P., James, R., Wiener, A., Mackenna, C. Law, D., York, A., Jones, M., Fonagy, P., Fleming, I. y Munk, S. (2016). Thrive elaborated. CAMHS Press. https://www.annafreud. org/media/4817/thrive-elaborated-2nd-edition.pdf

Wolpert, M., Harris, R., Jones, M., Hodges, S., Fuggle, P., James, R., Wiener, A., McKenna, C., Law, D. y Fonagy, P. (2014). THRIVE: The AFC-Tavistock model for CAMHS. CAMHS Press. http://repository.tavistockandportman.ac.uk/id/eprint/941

Yeomans, F. F., Clarkin, J. F. y Kernberg, O. (2016). Psicoterapia Centrada en la Transferencia. Su aplicación al Trastorno Límite de la Personalidad. Desclée de Brouwer.

Artículo recibido: $16 / 02 / 2021$

Artículo aceptado: 10/06/2021 